\title{
Critical role of acute hypoxemia on the cognitive impairment after severe COVID-19 pneumonia: a multivariate causality model analysis
}

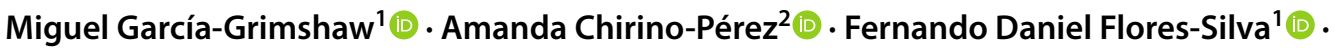 \\ Sergio Iván Valdés-Ferrer ${ }^{1,3}$ - María de los Ángeles Vargas-Martínez ${ }^{1}$. Ana Itiel Jiménez-Ávila ${ }^{1}$. \\ Oswaldo Alan Chávez-Martínez ${ }^{1}$. Enrique Manuel Ramos-Galicia ${ }^{4}$. Osvaldo Alexis Marché-Fernández ${ }^{1}$. \\ Martha Fernanda Ramírez-Carrillo ${ }^{4}$ Samara Lissete Grajeda-González ${ }^{1}$. Marco Eduardo Ramírez-Jiménez ${ }^{1}$. \\ Emma Adriana Chávez-Manzanera ${ }^{5}$ (10) María Teresa Tusié-Luna ${ }^{6,7}$ (1) Ana Ochoa-Guzmán ${ }^{6}$ (1)

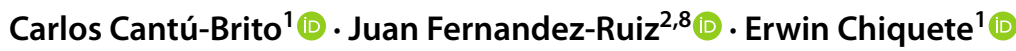

Received: 11 November 2021 / Accepted: 1 December 2021 / Published online: 13 January 2022

(c) Fondazione Società Italiana di Neurologia 2021

\begin{abstract}
Background A high proportion of coronavirus disease 2019 (COVID-19) survivors may develop long-term cognitive impairment. We aimed to develop a multivariate causal model exposing the links between COVID-19-associated biomarkers, illness-related variables, and their effects on cognitive performance.

Methods In this prospective study, we assess the potential drivers for the development of cognitive impairment in patients with severe COVID-19 pneumonia aged $\geq 18$ years at 6-month follow-up after hospital discharge, using the Montreal Cognitive Assessment (MoCA). Patients with pre-existing cognitive impairment were excluded. Laboratory results at hospital admission were clustered by principal component analysis (PCA) and included in a path analysis model evaluating the causal relationship between age, comorbidities, hypoxemia, invasive mechanical ventilation (IMV) requirement, in-hospital delirium, and cognitive performance.

Results We studied 92 patients: 54 (58.7\%) men and 38 (41.3\%) women, with median age of 50 years (interquartile range 42-55), among whom 50 (54.4\%) tested positive for cognitive impairment at 6-month follow-up. Path analysis revealed a direct link between the thrombo-inflammatory component of PCA (C-reactive protein, fibrinogen, and neutrophils) and hypoxemia severity at hospital admission. Our model showed that low $\mathrm{PaO}_{2} / \mathrm{FiO}_{2}$ ratio values, unlike the thrombo-inflammatory component, had a direct effect on cognitive performance, independent from age, in-hospital delirium, and invasive mechanical ventilation.

Conclusion In this study, biomarkers of thrombo-inflammation in COVID-19 and low $\mathrm{PaO}_{2} / \mathrm{FiO}_{2}$ had a negative effect on cognitive performance 6 months after hospital discharge. These results highlight the critical role of hypoxemia as a driver for impaired cognition in the mid-term.
\end{abstract}

Keywords Cognition $\cdot$ Cognitive impairment $\cdot$ COVID-19 $\cdot$ Hospitalization $\cdot$ Inflammation $\cdot$ Outcome $\cdot$ SARS-CoV-2

Miguel García-Grimshaw, Amanda Chirino-Pérez and Fernando Daniel Flores-Silva contributed equally to this work as co-first authors.

Carlos Cantú-Brito, Juan Fernandez-Ruiz and Erwin Chiquete are senior co-authors

Extended author information available on the last page of the article

\section{Introduction}

Despite worldwide vaccination efforts against the severe acute respiratory syndrome coronavirus-2 (SARS-CoV-2), the ongoing coronavirus disease 2019 (COVID-19) pandemic continues to be a global public health problem [1]. Since the pandemic began, several acute and chronic neurologic and psychiatric manifestations of COVID-19 have been described [2-4]. As the long-term consequences of SARSCoV-2 infection are better understood, it has become evident 
that a high proportion of survivors may develop multiple long-term neuropsychiatric sequelae that may co-exist and reduce the quality of life for these patients, including cognitive dysfunction, depression, anxiety, and post-traumatic stress disorder (PTSD) [5-7].

Cognitive dysfunction is the most frequently reported long-term mental health disorder affecting up to half of the patients with severe COVID-19 6 months after hospital discharge [8]; despite this high frequency and the growing evidence of persistent cognitive deficits after SARS-CoV-2 infection, the pathogenetic mechanisms involved in their development are yet to be elucidated. Multiple studies addressing the underlying factors associated with the development of this sequela have shown conflicting results that may be partially related to different clinical and statistical approaches trying to understand the role of COVID-19-associated inflammatory response biomarkers or illness-related variables such as hypoxemia, invasive mechanical ventilation (IMV) requirements, delirium, and psychiatric sequelae as independent phenomena and not part of the same cascade of events [9-13]. In this regard, the building of multivariate causal models may contribute to clarify the pathogenic drivers of cognitive impairments after COVID-19.

Therefore, the present study aimed to develop a causal model by path analysis exposing the links between age, comorbidities, COVID-19-associated thrombo-inflammatory response biomarkers, hypoxemia severity at hospital admission, IMV requirements, in-hospital delirium, and their effect on cognitive performance among severe COVID-19 pneumonia survivors 6 months after hospital discharge.

\section{Materials and methods}

\section{Study design, setting, and patient selection}

For this prospective study, we recruited adult patients (age $\geq 18$ years) who were hospitalized for severe COVID19 pneumonia according to the World Health Organization case definition [14], between March 15 and June 15, 2020, at the Instituto Nacional de Ciencias Médicas y Nutrición Salvador Zubirán, a tertiary-care hospital in Mexico City converted into a COVID-19 referral center. Following conversion, COVID-19-specific diagnostic and care protocols were implemented. Due to the potential risk of viral aerosolization, all patients under IMV were admitted to the intensive care unit (ICU) or ICU-adapted areas, while non-IMV patients were treated in general medical wards; when ICU beds or adapted areas were not available, patients requiring IMV were transferred to other hospitals [15]. The study protocol was revised and approved by our Institutional Research and Ethics Committees (reference: NER-3497-20-21-1). Signed informed consent was obtained from all participants and the procedures about human research were according to the Helsinki declaration.

As part of an ongoing study on long-term neuropsychiatric complications of COVID-19, cognitive and psychiatric assessments were performed at a follow-up visit scheduled 6 months after hospital discharge by a neuropsychologist, a psychiatrist, and a neurologist. We included patients with confirmed SARS-CoV-2 infection by real-time reverse transcription-polymerase chain reaction in respiratory specimens from nasopharyngeal swabs and COVID-19-compatible findings on chest computed tomography scan. None of the included patients received corticosteroids because they were treated before the preliminary report of their benefits in severe COVID-19 [16]. For this analysis, we excluded patients with a history of pre-existing cognitive impairment or other diseases associated with cognitive decline, as well as those who developed an in-hospital neurologic complication potentially associated with long-term cognitive impairment.

\section{Cognitive and psychiatric assessment}

Cognitive and psychiatric evaluations were performed using Spanish versions of screening instruments validated for the Mexican population. Cognitive assessment was performed using the Montreal Cognitive Assessment (MoCA), a screening instrument evaluating the following domains: visuospatial/executive ( 5 points), naming ( 3 points), memory ( 5 points), attention ( 6 points), language ( 3 points), abstraction ( 2 points), and orientation (6 points), whit a total score of 30 points. After correcting for years of education by adding an extra point to the total score for those cases with $\leq 12$ years of schooling, a score of $\leq 26$ points was considered a positive screening [17]. In addition, the Short Form of the Informant Questionnaire on Cognitive Decline in the Elderly (S-IQCODE), a 16-item screening tool for pre-existing cognitive impairment, was used in patients aged $\geq 50$ years; an adjusted score $\geq 3.4$ was considered positive [18].

Anxiety and depression assessments were performed using the Hospital Anxiety and Depression Scale (HADS), a 14-item scale with two subscales (seven items each), one for depression (HADS-D) and another for anxiety (HADS-A), scored on a Likert scale ranging from 0 (lowest) to 3 (highest). A cutoff of $\geq 11$ points for each disorder subscale was considered a positive screening [19, 20]. PTSD was assessed with the PTSD Checklist (PCL-5), a 20-item screening scale, scored on a Likert scale ranging from 0 (lowest) to 4 (highest); a cutoff of $>33$ points subscale was considered positive [21].

\section{Data collection and definitions}

Retrospective data from hospitalization were extracted from electronic medical records using a standardized case report 
format and entered into a secure online database, including demographics (age and sex), number of comorbidities (diabetes, hypertension, cardiovascular disease, pulmonary disease, current smoking, and obesity), and self-reported COVID-19-associated symptoms; the following blood workup results at admission: white blood cell count, arterial blood gas (ABG) analysis, lactate dehydrogenase, thrombotic, and inflammatory response biomarkers (D-dimer, fibrinogen, ferritin, and C-reactive protein); all results were interpreted according to our local laboratory reference limits. Treatment with vasopressors, opioids, benzodiazepines, or propofol as ever used; IMV requirements, its duration in days, and hospital length of stay; the development of delirium during hospitalization as either present or absent, delirium diagnostic protocols during the pandemic for each hospital setting in our center have been published elsewhere [15].

For each patient, we calculated the Charlson Comorbidity Index [22]. The partial pressure of arterial oxygen/fraction of inspired oxygen $\left(\mathrm{PaO}_{2} / \mathrm{FiO}_{2}\right)$ ratio on admission (before IMV) was calculated using the estimated $\mathrm{FiO}_{2}$ provided by each oxygen delivery device (nasal cannula or simple facemask) when the first blood samples for $\mathrm{ABG}$ analysis were obtained. Hypoxemia was defined as a $\mathrm{PaO}_{2} / \mathrm{FiO}_{2}$ ratio $\leq 300 \mathrm{mmHg}$, and its severity was classified as mild $\left(\mathrm{PaO}_{2} / \mathrm{FiO}_{2}\right.$ between 300 and $\left.201 \mathrm{mmHg}\right)$, moderate $\left(\mathrm{PaO}_{2} /\right.$ $\mathrm{FiO}_{2}$ between 200 and $\left.101 \mathrm{mmHg}\right)$, and severe $\left(\mathrm{PaO}_{2} / \mathrm{FiO}_{2}\right.$ ratio $\leq 100 \mathrm{mmHg}$ ) according to the Berlin definition cutoff values for acute respiratory distress syndrome (ARDS) [23]. On admission, the mortality risk was estimated with the Sequential Organ Failure Assessment (SOFA) score [24]. At least two researchers reviewed all data, and a third researcher adjudicated any difference in interpretation between the primary reviewers.

\section{Statistical analysis}

Categorical variables are reported as frequencies with proportions, and continuous variables as either median with interquartile range (IQR) or mean with standard deviation (SD). We compared the demographic characteristics, comorbidities, COVID-19-associated symptoms, and in-hospital events between patients with a positive screening for cognitive impairment and those with a negative assessment. After testing normality with the Kolmogorov-Smirnov test, continuous variables were analyzed with the Mann-Whitney $U$ test or Student's $t$ test, and as for categorical variables with the $\chi^{2}$ or Fisher's exact tests; the effect size for each test was evaluated by Cohen's $d, r$ scores, or Cramer's $V$, as appropriate.

One-sample $t$ tests were conducted to examine if mean measurements from laboratory findings were higher than their upper reference limit and if mean MoCA scores were lower than their clinical cutoff value. Bootstrapping with 10,000 samples was performed to avoid the assumption of normality. The effect size was evaluated by Cohen's $d$. Bivariate correlation analyses were conducted with Pearson or Spearman's rho correlation coefficient, as appropriate. MoCA total scores were compared among patients with and without hypoxemia using the Mann-Whitney $U$ test. Comparisons between hypoxemia severity regarding total MoCA scores were conducted with the Kruskal-Wallis test, and the effect size was calculated with $\varepsilon^{2}$. These analyses were performed using IBM SPSS Statistics, version 25 (IBM Corp., Armonk, NY, USA). Multiple comparison/correlation problem was addressed by False Discovery Rate corrections $(q$ value $=0.05)$.

To explore the dimension underlying the laboratory findings, we conducted a principal component analysis (PCA) using MATLAB, version 2018b (MathWorks Inc., Natick, MA, USA). Before PCA, C-reactive protein, D-dimer, LDH, ferritin, fibrinogen, neutrophils, and lymphocytes measurements were converted to $z$-scores based on the SD of the cohort. Considering the pathophysiology of COVID-19, lymphocytes measures were inverted, ensuring that lower values would indicate worse disease severity [25]. Components with eigenvalues $\geq 1$ were extracted and varimaxrotated, yielding orthogonal results. Scores from each principal component were identified. Each component was interpreted according to the current understanding of the COVID-19 pathophysiology [26, 27].

To simultaneously test the relationship between the components obtained from the PCA, age, number of comorbidities, $\mathrm{PaO}_{2} / \mathrm{FiO}_{2}$ ratio, IMV duration, in-hospital delirium, and cognitive performance, a causation structure between these variables was modeled by path analysis using IBM SPSS Amos 24 (IBM Corp., Armonk, NY, USA). Before model testing, Mardia's test was conducted to assess multivariate normality according to its skewness and kurtosis [28]. Due to multivariate normality violations, bootstrapping was performed (10,000 samples) to correct the $P$ values from the $\chi^{2}$ tests and estimate direct and indirect effects (95\% confidence intervals) using the maximum likelihood method [29]. Each observed variable had a measurement error associated.

Based on the first hypothetical model results, non-significant paths with low standardized beta values were removed to promote parsimony and a good model fit evaluated with the following indexes: comparative fit index (CFI), root mean square error approximation (RMSEA), goodness of fit index (GFI), parsimony-adjusted normed fit index (PNFI), $\chi^{2}$ (degrees of freedom $[d f]$ ), and $\chi^{2} / d f$ ratio. After identifying significant direct effects from the final model, we calculated indirect effects. For all analyses, $P$ values were two-tailed and considered significant when $<0.05$. 


\section{Results}

\section{Demographic and clinical data}

In total, 113 patients completed the cognitive assessment; of them, we excluded 21 (18.6\%) from the final analysis: 18 $(85.7 \%)$ due to a history of the following diseases associated with cognitive decline: hypothyroidism in six; epilepsy in three (one of them an HIV-positive patient); major depressive disorder in three; and chemotherapy-treated oncologic disease, ischemic stroke, and pre-existing cognitive impairment two cases each; three (14.3\%) due to the following in-hospital events: acute ischemic stroke in two and newonset seizures requiring long-term antiepileptic drugs in one. None of the included patients tested positive for pre-existing cognitive impairment.

We studied 92 patients, 54 (58.7\%) males and 38 (41.3\%) females, with a median age of 50 (IQR 42-55) years; 50 (54.4\%) tested positive for cognitive impairment; patient's baseline characteristics are reported in Table 1. One-sample $t$ tests comparing the laboratory findings with their respective upper reference limits showed that our patients had higher values in all tests except for neutrophil counts (Fig. 1A); lymphocyte values were lower than their inferior reference limit. The overall mean MoCA total scores were lower than its clinical cutoff value ( $\leq 26$ points). There were no differences in age, comorbidities, self-reported symptoms, or inhospital events between patients who tested positive for cognitive impairment and those who did not (Table 1). Patients with a positive MoCA exhibited significantly lower $\mathrm{PaO}_{2} /$ $\mathrm{FiO}_{2}$ ratio values $(U=794.5, P=0.045, r=-0.21)$. The differences between MoCA total and subset scores between groups are reported in Table 2.

Due to protocol amendments, only 67 (72.7\%) patients completed the psychiatric evaluations; three $(4.5 \%)$ tested positive for depression, 15 (22.4\%) for anxiety, and 12 (17.9\%) for both, and 13 (19.4\%) for PTSD, of whom only one patient had a negative HADS screening. There were no statistical differences between patients with cognitive impairment $(n=38,56.7 \%)$ and those without $(n=29$, $43.3 \%$ ) in both positivity (Table S1) or scores (Table S2) for each of these psychiatric assessments. In this regard, neither HADS (anxiety: $\rho=-0.02, P=0.863$; depression: $\rho=-0.11, P=0.386$; total score: $\rho=-0.08, P=0.514$ ) nor PCL5 ( $\rho=-0.18, P=0.149)$ scores showed significant correlations with total MoCA scores.

\section{Principal component analysis of COVID-19-associated biomarkers}

PCA exploring the studied biomarkers produced a three orthogonal component solution accounting for $70.13 \%$ of the variance (Kaiser-Meyer-Olkin index $=0.74$ ). Measurements loading highest on each component $(>0.4)$ are shown in Fig. 1B. The first component (accounting for $39.73 \%$ of the variance) was interpreted as "thrombo-inflammatory" which included the following biomarkers: C-reactive protein, fibrinogen, and neutrophils. The second component (accounting for $16.43 \%$ of the variance) loaded heavily to D-dimer and LDH; hence, this component was interpreted as "thrombosis-related tissue damage." Finally, the third component (accounting for $13.97 \%$ of the variance) was interpreted as "acute inflammation," as lymphocytes and ferritin loaded heavily.

\section{Causal model for cognitive impairment}

Correlations between the "thrombo-inflammatory" component, hypoxemia severity, days of IMV, in-hospital delirium, age, and cognitive impairment are shown in Fig. 1C. Neither scores from the "thrombosis-related tissue damage" component nor the "acute inflammation" component showed significant correlations with the variables included in the path model (Table S3). Regarding path analysis, the hypothesized model provided an adequate fit for the data, but a poor parsimony (Fig. 2A). The model fit statistics were as follows: $\mathrm{CFI}=0.99, \mathrm{TLI}=0.98$, RMSEA $=0.03$, GFI $=0.97$, and PNFI $=0.33 \cdot \chi^{2}(d f)$ value was 14.20 (13) with $P=0.360$ and $P_{\text {bootstrapped }}=0.609$. $\chi^{2} / d f$ ratio was 1.09 . To improve parsimony, non-significant paths with low standardized $\beta$ values were removed. These adjustments resulted in a final model well fitted to our data (Fig. 2B) according to the following fit indexes: $\mathrm{CFI}=0.98, \mathrm{TLI}=0.97, \mathrm{RMSEA}=0.05, \mathrm{GFI}=0.95$, and $\mathrm{PNFI}=0.64 . \chi^{2}(d f)$ value was $17.99(15)$ with $P=0.263$ and $P_{\text {bootstrapped }}=0.202 . \chi^{2} / d f$ ratio was 1.20 .

\section{Cognitive performance}

A positive direct relationship was found between $\mathrm{PaO}_{2} / \mathrm{FiO}_{2}$ values at hospital admission and cognitive performance $(\beta=0.24, P=0.010)$. Also, age negatively impacted cognitive performance $(\beta=-0.18, P=0.052)$. Neither days of $\operatorname{IMV}(\beta=0.16, P=0.403)$ nor in-hospital delirium $(\beta=0.03$, $P=0.954)$ had direct effects on MoCA's total score. On our hypothesized model, we did not find that MoCA's total score had a direct impact from the thrombo-inflammatory component $(\beta=0.03, P=0.720)$, the thrombosis-related tissue damage component $(\beta=0.03, P=0.655)$, the acute inflammation component $(\beta=-0.08, P=0.726)$, or the number of comorbidities $(\beta=0.01, P=0.850)$. Nevertheless, the final model showed a significant indirect effect from the thromboinflammatory component to cognitive performance through $\mathrm{PaO}_{2} / \mathrm{FiO}_{2}$ values $(\beta=-0.10, P=0.007)$ (Table 3$)$. 
Table 1 Patients' baseline characteristics, self-reported COVID-19-associated symptoms, and events during hospitalization

\begin{tabular}{|c|c|c|c|}
\hline & All patients $(n=92)$ & Negative MoCA $(n=42)$ & Positive MoCA $(n=50)$ \\
\hline Age, median (IQR), years & $50(42-55)$ & $48(40-53)$ & $52(44-57)$ \\
\hline \multicolumn{4}{|l|}{ Sex, $n(\%)$} \\
\hline Female & $38(41.3)$ & $12(28.6)$ & $26(52)$ \\
\hline Male & $54(58.7)$ & $30(71.4)$ & $24(48)$ \\
\hline Years of education, mean $( \pm S D)$ & $13.4(4.3)$ & $14.7(4.4)$ & $12.3(4)$ \\
\hline \multicolumn{4}{|l|}{ Comorbidities, $n(\%)$} \\
\hline Diabetes & $19(20.7)$ & $10(23.8)$ & $9(18)$ \\
\hline Hypertension & $20(21.7)$ & $7(16.7)$ & $13(26)$ \\
\hline Cardiovascular disease & $4(4.3)$ & $4(9.5)$ & 0 \\
\hline Pulmonary disease & $4(4.3)$ & $1(2.4)$ & $3(6)$ \\
\hline Smoking & $7(7.6)$ & $3(7.1)$ & $4(8)$ \\
\hline Obesity & $55(59.8)$ & $26(61.9)$ & $29(58)$ \\
\hline \multicolumn{4}{|l|}{ Number of comorbidities, $n(\%)$} \\
\hline 1 & $44(44.6)$ & $21(50)$ & $20(40)$ \\
\hline 2 & $17(18.5)$ & $6(14.3)$ & $11(22)$ \\
\hline$\geq 3$ & $10(10.9)$ & $5(11.9)$ & $5(10)$ \\
\hline Charlson index, median (min-max) & $1(0-5)$ & $1(0-5)$ & $1(0-3)$ \\
\hline \multicolumn{4}{|l|}{ Reported symptoms, $n$ (\%) } \\
\hline Fever & $82(89.1)$ & $37(88.1)$ & $45(90)$ \\
\hline Anosmia & $13(14.1)$ & $6(14.3)$ & $7(14)$ \\
\hline Dysgeusia & $11(12)$ & $5(11.9)$ & $6(12)$ \\
\hline Headache & $50(54.3)$ & $22(52.4)$ & $28(56)$ \\
\hline Muscle pain & $44(47.8)$ & $20(47.6)$ & $24(48)$ \\
\hline Dyspnea & $74(80.4)$ & $32(76.2)$ & $42(84)$ \\
\hline Cough & $73(79.3)$ & $39(92.9)$ & $34(68)$ \\
\hline Diarrhea & $16(17.4)$ & $10(23.8)$ & $6(12)$ \\
\hline $\mathrm{PaO}_{2} / \mathrm{FiO}_{2}$ ratio, median (IQR), $\mathrm{mmHg}$ & $214(162-290)$ & $245(179-337)$ & $198(160-247)$ \\
\hline \multicolumn{4}{|l|}{ Hypoxemia severity, $n(\%)$} \\
\hline Mild & $31(33.7)$ & $14(33.3)$ & $17(34)$ \\
\hline Moderate & $36(39.1)$ & $11(26.2)$ & $25(50)$ \\
\hline Severe & $4(4.3)$ & $3(7.1)$ & $1(2)$ \\
\hline IMV requirement, $n(\%)$ & $16(17.4)$ & $8(19)$ & $8(16)$ \\
\hline Duration of IMV, median (IQR), days & $12(8.5-20)$ & $15.5(10-22)$ & $11.5(5.5-13.5)$ \\
\hline Delirium, $n(\%)$ & $13(14.1)$ & $8(19)$ & $5(10)$ \\
\hline Vasopressor support, $n(\%)$ & $14(15.2)$ & $8(19)$ & $6(12)$ \\
\hline Propofol treatment, $n(\%)$ & $14(15.2)$ & $7(16.7)$ & $7(14)$ \\
\hline Opioids treatment, $n(\%)$ & $16(17.4)$ & $8(19)$ & $8(16)$ \\
\hline Benzodiazepine treatment, $n(\%)$ & $16(17.4)$ & $8(19)$ & $8(16)$ \\
\hline
\end{tabular}

$\mathrm{SD}$, standard deviation; min, minimum; max, maximum; $\mathrm{IQR}$, interquartile range; $\mathrm{PaO}_{2} / \mathrm{FiO}_{2}$, partial pressure of arterial oxygen/fraction of inspired oxygen; $I M V$, invasive mechanical ventilation. Vasopressor support, propofol, opioids, and benzodiazepines are reported as ever used

\section{In-hospital delirium}

The number of days under IMV was positively and significantly associated with in-hospital delirium $(\beta=0.84$, $P=0.001)$, indicating that patients with longer days under IMV were more likely to develop delirium during their hospital stay. We found no direct relationship between $\mathrm{PaO}_{2} / \mathrm{FiO}_{2}$ values and delirium $(\beta=-0.01$, $P=0.740$ ). Table 3 shows a significant indirect effect from the thrombo-inflammatory component to in-hospital delirium through $\mathrm{PaO}_{2} / \mathrm{FiO}_{2}$ values and IMV duration ( $\beta=0.10, P=0.005)$. IMV duration was also a significant mediator variable between $\mathrm{PaO}_{2} / \mathrm{FiO}_{2}$ values and in-hospital delirium $(\beta=-0.24, P=0.007)$. 


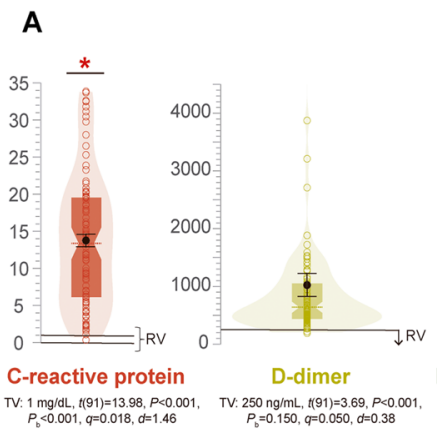

B

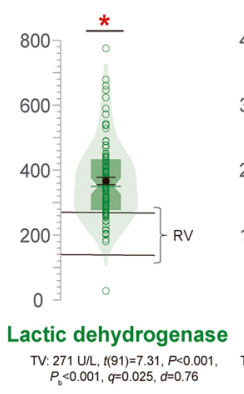

TV: 271 U/L, $(191)=7.31, P<0.001$

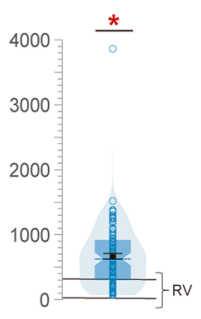

Ferritin

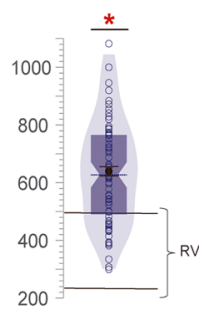

Fibrinogen

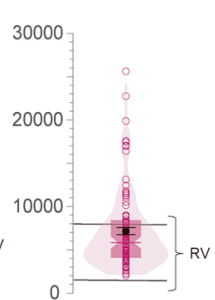

Neutrophils

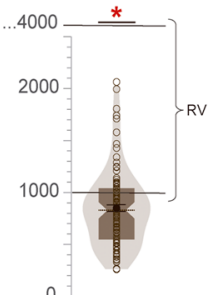

0

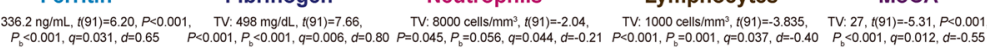

C
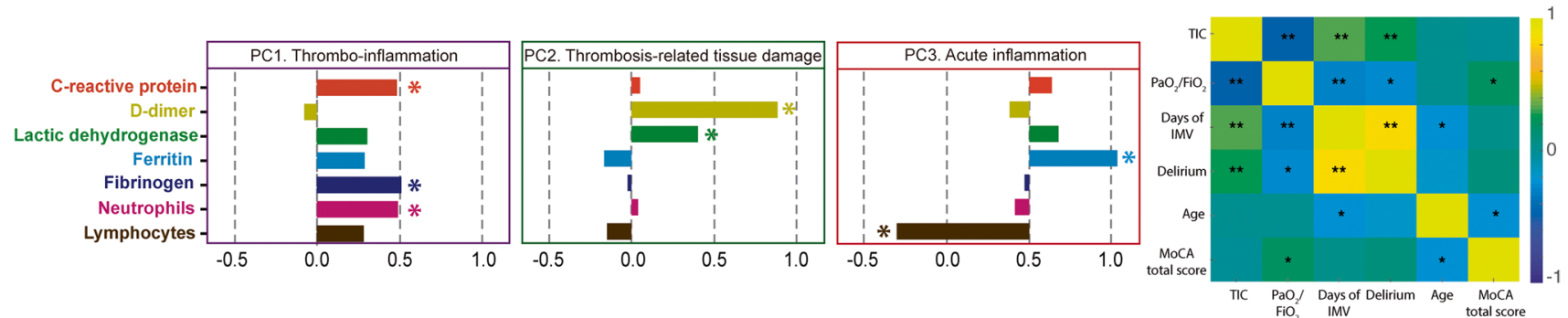

Fig. 1 Laboratory findings in COVID-19 and correlations between illness-related variables. A Comparative analyses between the measurements derived from the blood workups and their upper reference limit (lower reference limit for lymphocytes); and comparison between MoCA total score and its clinical cutoff point. The shadows in the violin plots show the probability density of the data smoothed by a kernel density estimator. The boxes inside the shadows represent the first and third quartile and the median in a dotted line. The superior and inferior notch in the boxes point out the lower and upper bounds of the $95 \%$ confidence interval for the median. The black points represent the mean, and the bars show the standard error of the mean. ${ }^{*} P<0.05$ after False Discovery Rate corrections $(q=0.05)$. B Loadings of the components derived from the PCA. *Biomarkers loading highest $(>0.4)$ at each component. C Correlations among study variables included in the path analysis. $* P<0.05, * * P<0.01$. MoCA, Montreal Cognitive Assessment; $P_{\mathrm{b}}, P$ bootstrapped $(10,000$ iterations); TIC, thrombo-inflammatory component; IMV, invasive mechanical ventilation; $\mathrm{PaO}_{2} / \mathrm{FiO}_{2}$, partial pressure of arterial oxygen/fraction of inspired oxygen ratio; $\mathrm{TV}$, test value; $\mathrm{RV}$, reference value

Table 2 Montreal Cognitive Assessment total and subset scores according to positivity

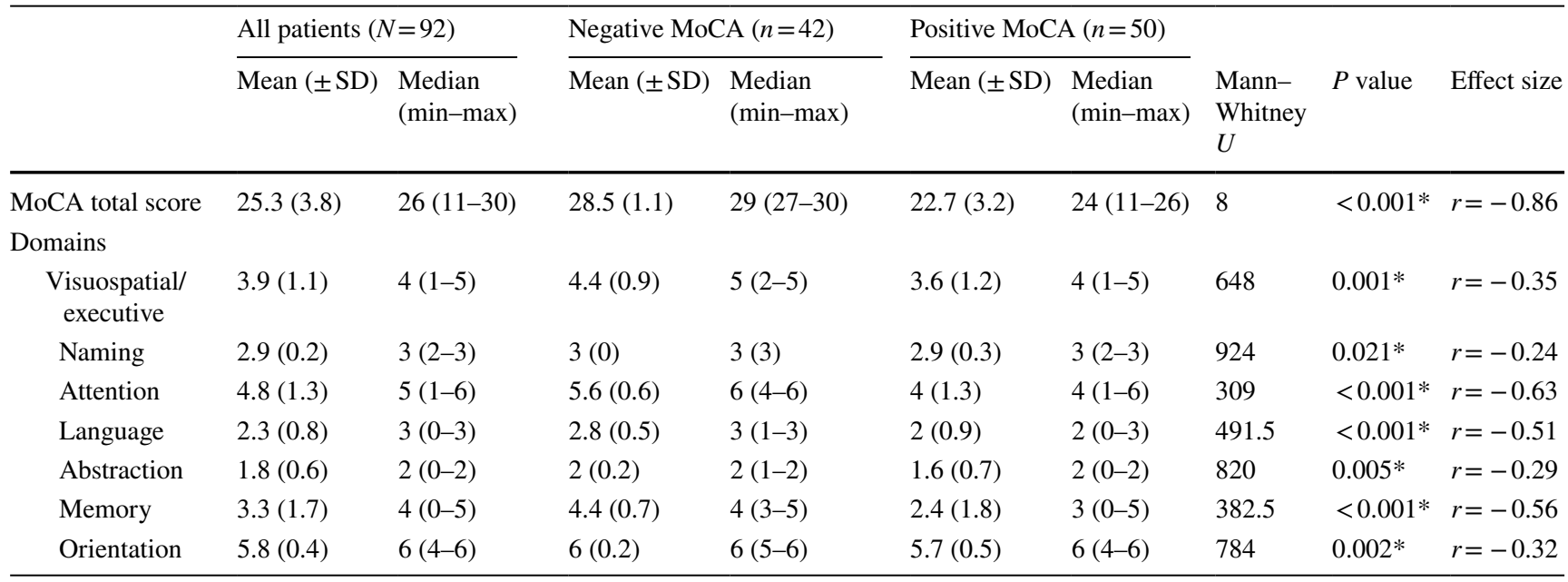

MoCA, Montreal Cognitive Assessment; $S D$, standard deviation, Min, minimum; Max, maximum. *Significant $P$ value after False Discovery Rate correction 


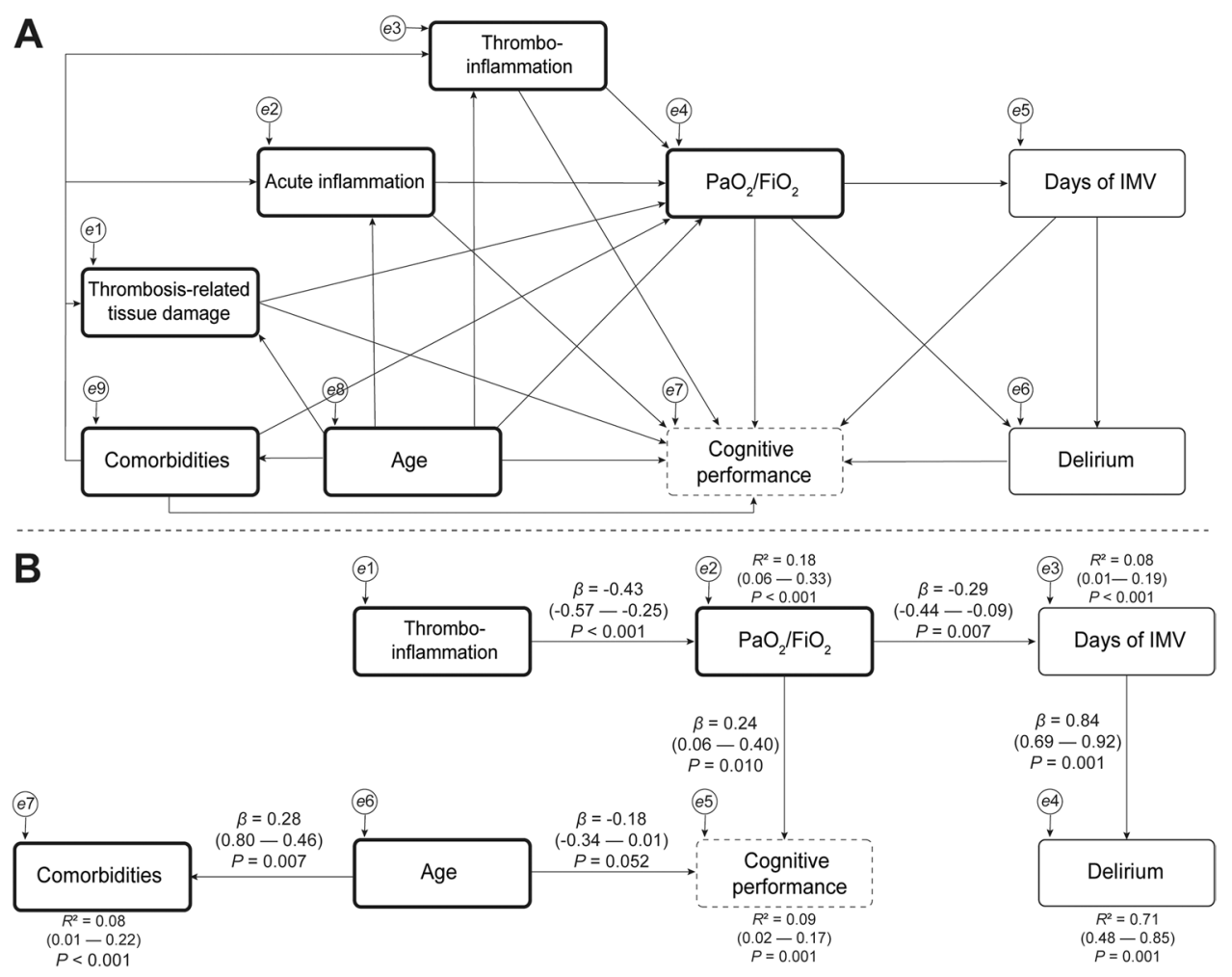

Fig. 2 Results of the path analysis on factors associated with cognitive performance. A Hypothetical path model. B Final path model presents significant causal relationships. The arrows represent an association between variables. Standardized coefficients $(\beta), 95 \%$ confidence intervals, and associated $P$ values are shown above each arrow. The boxes represent observable variables and their measurement errors $(e)$. Boxes with thick solid lines are variables acquired at hospital admission, boxes with thin solid lines are in-hospital events, and the box with dotted lines represents cognitive performance measured by the Montreal Cognitive Assessment 6 months after hospital discharge. The proportion of variance explained $\left(R^{2}\right), 95 \%$ confidence intervals, and associated $P$ values are shown above and below endogenous variables. The number of comorbidities included diabetes, hypertension, cardiovascular disease, pulmonary disease, smoking, and obesity. $\mathrm{PaO}_{2} / \mathrm{FiO}_{2}$, partial pressure of arterial oxygen/fraction of inspired oxygen ratio; IMV, invasive mechanical ventilation
Table 3 Indirect effects for the final path model

\begin{tabular}{|c|c|c|c|c|}
\hline \multirow[t]{2}{*}{ Indirect effects } & \multirow{2}{*}{$\begin{array}{l}\text { Standardized } \\
\text { coefficients }(\beta)\end{array}$} & \multirow[t]{2}{*}{$P$ value } & \multicolumn{2}{|c|}{ 95\% Confidence interval* } \\
\hline & & & Lower limit & Upper limit \\
\hline $\mathrm{TIC} \rightarrow \mathrm{PaO}_{2} / \mathrm{FiO}_{2}$ ratio $\rightarrow$ Days of IMV & 0.12 & .005 & 0.04 & 0.23 \\
\hline $\mathrm{TIC} \rightarrow \mathrm{PaO}_{2} / \mathrm{FiO}_{2}$ ratio $\rightarrow$ Days of IMV $\rightarrow$ Delirium & 0.10 & .005 & 0.03 & 0.20 \\
\hline $\mathrm{PaO}_{2} / \mathrm{FiO}_{2}$ ratio $\rightarrow$ Days of IMV $\rightarrow$ Delirium & -0.24 & .007 & -0.39 & -0.08 \\
\hline $\mathrm{TIC} \rightarrow \mathrm{PaO}_{2} / \mathrm{FiO}_{2}$ ratio $\rightarrow$ Cognitive performance & -0.10 & .007 & -0.20 & -0.03 \\
\hline
\end{tabular}

$T I C$, thrombo-inflammatory component; $\mathrm{PaO}_{2} / \mathrm{FiO}_{2}$, partial pressure of arterial oxygen/fraction of inspired oxygen; $I M V$, invasive mechanical ventilation. *Bootstrapped confidence intervals

\section{Invasive mechanical ventilation duration and $\mathrm{PaO}_{2} /$ $\mathrm{FiO}_{2}$ ratio}

$\mathrm{PaO}_{2} / \mathrm{FiO}_{2}$ values were directly and negatively related with the number of days on IMV $(\beta=-0.29$, $P=0.007)$. Also, we found a significant indirect effect from the thrombo-inflammatory component to days of IMV through $\mathrm{PaO}_{2} / \mathrm{FiO}_{2}$ values $(\beta=0.12, P=0.005$; Table 3). The thrombo-inflammatory component was negatively associated with $\mathrm{PaO}_{2} / \mathrm{FiO}_{2}$ values $(\beta=-0.43$, $P<0.001)$. Levels of $\mathrm{C}$-reactive protein, neutrophils, and fibrinogen, which loaded heavily on this component, showed significant negative correlations with $\mathrm{PaO}_{2} / \mathrm{FiO}_{2}$ values (Fig. 3A, B, and C). In contrast, neither the thrombosis-related tissue damage component ( $\beta=-0.15, P=0.196)$ nor the acute inflammation component $(\beta=0.05, P=0.490)$ was directly associated with $\mathrm{PaO}_{2} / \mathrm{FiO}_{2}$ values. 
A

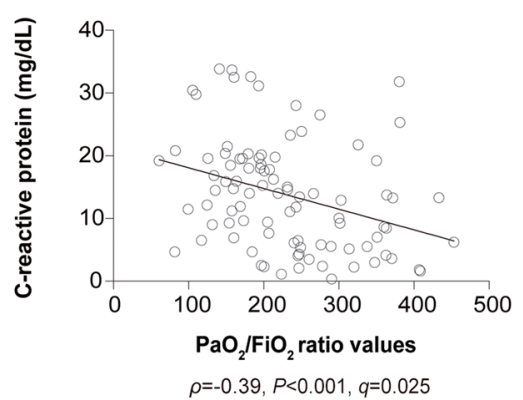

D

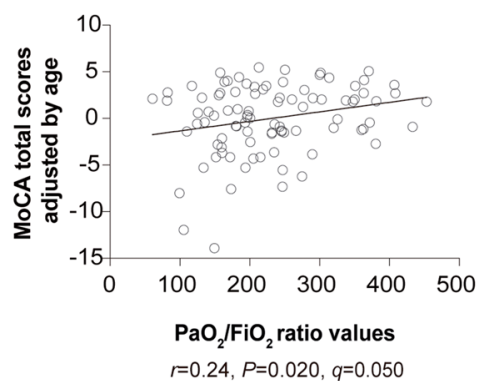

B

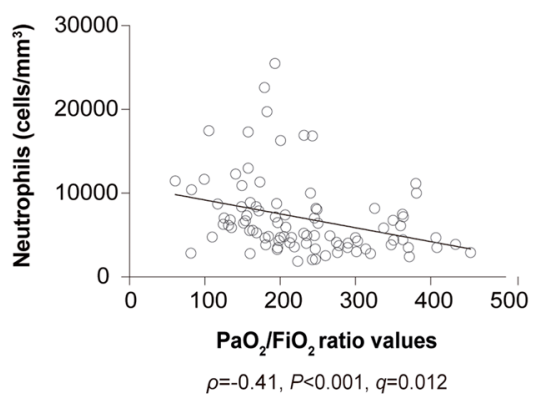

E

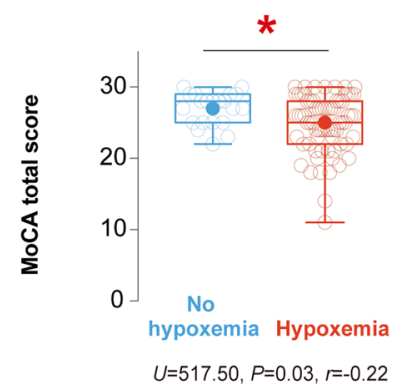

C
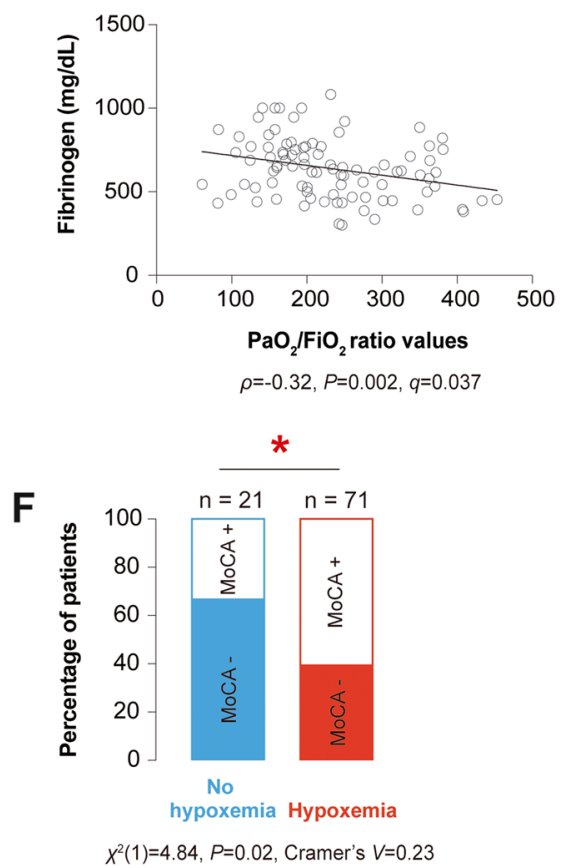

Fig. 3 Association of hypoxemia and cognitive impairment. Significant relationships between $\mathrm{PaO}_{2} / \mathrm{FiO}_{2}$ ratio values and A C-reactive protein, $\mathbf{B}$ neutrophils, and $\mathbf{C}$ fibrinogen. D Semipartial correlation between $\mathrm{PaO}_{2} / \mathrm{FiO}_{2}$ values at hospital admission and MoCA's total scores adjusted by age. The $Y$-axis is expressed as residuals. E Comparative analysis of MoCA performance between patients with $\left(\mathrm{PaO}_{2} /\right.$ $\left.\mathrm{FiO}_{2} \leq 300 \mathrm{mmHg}\right)$ and without $\left(\mathrm{PaO}_{2} / \mathrm{FiO}_{2}>300 \mathrm{mmHg}\right)$ hypoxemia

\section{COVID-19 biomarkers}

Age was positively and significantly associated with the number of comorbidities $(\beta=0.28, P=0.007)$; however, neither of these two variables showed significant relationships with $\mathrm{PaO}_{2} / \mathrm{FiO}_{2}$ values (age: $\beta=-0.04, P=0.808$; comorbidities: $\beta=0.11, P=0.320$ ), the thrombo-inflammatory component (age: $\beta=-0.01, P=0.872$; comorbidities: $\beta=-0.01, P=0.942$ ), the thrombosis-related tissue damage component (age: $\beta=-0.18, P=0.101$; comorbidities: $\beta=-0.03, P=0.799$ ), or the acute inflammation component (age: $\beta=0.13, P=0.211$; comorbidities: $\beta=-0.27$, $P=0.050)$.

\section{Causal model for MoCA subtests}

The causal model exposed in Fig. 2B (including MoCA total score as endogenous/outcome variable) also explained the performance in the visuospatial/executive and verbal memory subsets (Supplementary Fig. 1); for both, the model had an acceptable fitting with the data. The model including scores from MoCA's visuospatial/executive subtest as endogenous/outcome variable at hospital admission. The solid point inside the boxes represents the mean, whiskers the minimum and maximum values. F Proportion of positive $(\mathrm{MoCA}+)$ and negative $(\mathrm{MoCA}-)$ screening for cognitive impairment (clinical cutoff point $\leq 26$ ) among patients with and without hypoxemia at hospital admission. $\mathrm{PaO}_{2} / \mathrm{FiO}_{2}$, partial pressure of arterial oxygen/fraction of inspired oxygen ratio; MoCA, Montreal Cognitive Assessment

yielded the following fit indexes: $\mathrm{CFI}=0.99$; $\mathrm{TLI}=0.99$; RMSEA $=0.02 ;$ GFI $=0.96 ;$ PNFI $=0.65 ; \chi^{2}(d f)=15.56$ (15), $P=0.412, P_{\text {bootstrapped }}=0.350 ; \chi^{2} / d f=1.04$; while fit indexes for the model which included MoCA's verbal memory scores were as follows: $\mathrm{CFI}=0.99$; $\mathrm{TLI}=0.99$; $\mathrm{RMSEA}=0.03 ; \mathrm{GFI}=0.95 ; \mathrm{PNFI}=0.64 ; \chi^{2}(d f)=16.38$ (15), $P=0.357, P_{\text {bootstrapped }}=0.305 ; \chi^{2} / d f=1.09$.

As in the first model, $\mathrm{PaO}_{2} / \mathrm{FiO}_{2}$ values were directly and positively related with the performance on visuospatial/ executive $(\beta=0.26, P=0.017)$ and verbal memory $(\beta=0.20$, $P=0.020)$ subsets, with significant indirect effects between thrombo-inflammatory component and performance in both subtests through $\mathrm{PaO}_{2} / \mathrm{FiO}_{2}$ values (visuospatial/executive: $\beta=-0.11, P=0.013$; verbal memory: $\beta=-0.09$, $P=0.015)$.

\section{Hypoxemia and cognitive performance}

We regressed out age from MoCA total scores and explored their correlation with $\mathrm{PaO}_{2} / \mathrm{FiO}_{2}$ values based on the path analysis results. As predicted by the model, MoCA scores adjusted by age significantly correlated with $\mathrm{PaO}_{2} / \mathrm{FiO}_{2}$ values (Fig. 3D). We compared the cognitive performance 
between patients with $(n=71,77.2 \%)$ and without $(n=21$, $22.8 \%$ ) hypoxemia. There were no differences in sex, age, years of education, and psychiatric features between groups (Table S4). As shown in Fig. 3E, patients with hypoxemia showed significantly lower MoCA scores than patients without hypoxemia. Furthermore, a larger number of patients with hypoxemia (60.6\%) had scores below MoCA's clinical cutoff point compared to those without hypoxemia (33.3\%) (Fig. 3F).

MoCA total scores did not show significant differences ( $H=0.78$, asymptotic $P=0.68$, Monte Carlo $P=0.69$, $\left.\varepsilon^{2}=0.01\right)$ between patients with mild $(n=31$, median 25 , IQR $22-29$ points), moderate ( $n=36$, median 25.5 , IQR $22.5-27.5$ points), or severe ( $n=4$, median 27.5 , IQR $22.5-28$ points) hypoxemia. Neither demographic nor psychiatric features showed significant differences among these three groups (Table S5). When including the four severely hypoxemic patients in the moderate group and comparing their cognitive performance with the mild group, the same non-significant results were found $(U=588.50, P=0.714$, $r=-0.04)$. Regarding the number of patients with a positive MoCA, there was no statistical difference $\left(\chi^{2}(1)=0.75\right.$, $P=0.38$, Cramer's $V=0.10$ ) between patients with mild (54.8\%) and moderate/severe (65\%) hypoxemia.

\section{Discussion}

In this interim analysis of an ongoing study on the long-term neuropsychiatric consequences of SARS-CoV-2 infection, the frequency of new-onset cognitive impairment 6 months after hospital discharge for severe COVID-19 pneumonia was $54.4 \%$, similar to that of $52.6 \%$ reported by Frontera et al., in a cohort of 382 patients evaluated 6 months after hospital discharge, assessed with a MoCA telephone version [8]. Even though their population was older (median age 68 years, IQR 55-77) than ours and had a higher burden of neurologic and non-neurologic comorbidities that can negatively affect cognition [30-32], in our population, neither age nor comorbidities were associated with worse MoCA scores.

Among critically ill non-COVID-19 patients, IMV is independently associated with poor performance on cognitive evaluations 6 months after hospital discharge [33, 34]; also, prolonged IMV exposing them to higher cumulative doses of sedatives or opioids increases the risk of developing in-hospital delirium [35, 36], a well-recognized risk factor for long-term cognitive impairment after hospitalization [37]. Among hospitalized patients with COVID-19, delirium incidence ranges from 11 to $80 \%$ depending on the hospital setting (general ward or ICU) [38, 39]; in our population, we previously reported a frequency of $16.3 \%$ regardless of the hospital setting [15], a proportion comparable to the $14.1 \%$ of patients included in this study who developed this complication. As reported by other authors in COVID-19 [9, 40], we did not find a direct or indirect effect between IMV duration, in-hospital delirium, and cognitive performance.

In our structural approach, the thrombo-inflammatory component (C-reactive protein, fibrinogen, and neutrophils) obtained from the PCA indirectly affected cognition, an effect mediated through $\mathrm{PaO}_{2} / \mathrm{FiO}_{2}$ ratio values. Despite this, there was no association between hypoxemia severity according to the Berlin definition cutoff values for ARDS and total MoCA scores on univariate analysis. In the study conducted by Frontera et al., low oxygen levels or the severity of lung injury was not associated with worse cognitive outcomes [8], as seen in non-COVID-19 critically ill patients $[33,41]$; these contrasting results may be related to different methodological and statistical approaches. Interestingly, a case series of 29 patients found an association between residual pulmonary dysfunction assessed 3-4 months after hospital discharge and cognitive impairment [42], suggesting that long-lasting cerebral oxygen delivery restriction is involved in developing this sequela.

Although structural neuroimaging studies were not performed in our patients, it is known that profound hypoxemia is associated with brain atrophy [43], especially in highly susceptible structures such as the hippocampus [44]; among COVID-19 patients, microstructural changes in this structure have been detected as early as 3 months after hospital discharge [45]. Therefore, we hypothesize that the vulnerability of this cognition-related structure to hypoxemia may explain the findings of impaired verbal memory, along with executive dysfunction seen in COVID-19 survivors [10, 46-48]. In our model, lower $\mathrm{PaO}_{2} / \mathrm{FiO}_{2}$ ratio values were also associated with deficits in the MoCA visuospatial/executive and verbal memory subsets, as well as MoCA total scores.

Besides hypoxemia, the hyperinflammatory and hypercoagulable states promoted by SARS-CoV-2 may also play a crucial role in developing cognitive deficits after COVID19 [47, 49]. In this regard, elevated D-dimer and C-reactive protein levels during hospitalization for severe COVID-19 correlated with worse cognitive performance in other studies $[42,50]$. We did not find individual statistical differences between these biomarkers by univariate analysis; however, when clustered by PCA, the thrombo-inflammatory component had an indirect effect on cognitive impairment. In a case-control study, elevated inflammatory response biomarkers (C-reactive protein, procalcitonin, and interleukin 6) among patients with COVID-19 correlated with microstructural changes of white matter tracts, decreased cortical thickness, and widespread cerebral blood flow reductions, especially in the frontal and limbic systems 3 months after discharge [51].

The aforementioned results support our hypothesis in COVID-19 structural brain changes of cognition-related 
structures, promoted by a combination of multiple factors, such as hypoxemia (acute or chronic), cerebrovascular endothelium dysfunction related to systemic inflammation, or microthrombosis, that may disrupt the blood-brain barrier facilitating the passage to the brain of pro-inflammatory molecules and cytokines which can cause microglial activation, axonopathy, and production of neurotoxic mediators leading to chronic impairment of neurotransmission [52, 53]. In addition, disruption of the blood-brain barrier can also facilitate the entry of SARS-CoV-2 to the brain [52].

Following hospitalization for severe COVID-19, psychiatric sequela associated with poor cognitive performance, including depression, anxiety, and PTSD, develops in up to $30 \%$ of patients [7, 54]. Although only $72.7 \%$ of our patients were evaluated for the co-existence of psychiatric disorders, we found no correlation between total MoCA scores and depression, anxiety, or PTSD scores, neither statistical differences according to each screening tool positivity. However, the results of studies analyzing the association between these sequelae and cognitive performance have yielded conflicting results [9-13], which may be related to the heterogeneity of screening or diagnostic instruments and different methodological approaches. These findings suggest that the long-term effects of COVID-19 on cognition are not entirely driven by psychiatric variables, probably due to their duration.

This study has limitations. First, the single-center design limits the generalizability of our findings; also, due to our sample size, we were unable to perform invariance analyses exploring our model fitness across sexes. Second, the potential uncontrolled confounding for variables such as delirium duration, in-hospital treatments that might have modified the clinical course of these patients, psychiatric comorbidities, the single measurement of biomarkers, or measurements of additional inflammatory biomarkers, such as in interleukins which were not available in our center. Third, the $\mathrm{PaO}_{2} / \mathrm{FiO}_{2}$ ratio in non-IMV patients may not be a reliable method to define hypoxemia. Fourth, our model results may be biased due to the low proportion of subjects who required IMV or developed in-hospital delirium; however, our patients had a lower burden of comorbidities associated with severity when compared to other similar series $[8,13]$. Fifth, we were unable to evaluate the potential neurotropism of SARS-CoV-2 to evaluate its role in developing this sequela. Finally, the lack of structural neuroimaging studies to analyze the relationship of brain changes with this COVID-19 sequela, as well as the administration of a single screening test to measure cognitive performance and not a more extensive battery of neuropsychological tests, limits the analysis of independent domains to characterize the specific patterns of cognitive impairment among severe COVID19 survivors. Despite these limitations, our statistical approach emphasizes the potential multicollinearity between hypoxemia, COVID-19-associated thromboinflammatory response biomarkers, and cognitive performance; the synergistic effects of these variables on cognition should be carefully considered by researchers seeking to characterize the pathogenic drivers of this COVID-19 sequela, particularly those studies conducting univariate approaches. Still, reports of poor cognitive performance among non-hospitalized patients with mild to moderate disease without oxygen requirement suggest that other factors besides hypoxemia are involved in developing this long-COVID manifestation $[10,55,56]$.

In conclusion, this study highlights the central role of hypoxemia and the thrombo-inflammatory response as drivers for developing cognitive impairment among patients with severe COVID-19 pneumonia. The remarkable high frequency of cognitive impairment 6 months after hospital discharge in this relatively young population with a low burden of comorbidities should emphasize the need for continuous neuropsychological monitoring for these patients, especially evaluating verbal memory and executive functions regardless of hypoxemia severity, in-hospital delirium development, IMV requirements, or results of follow-up psychiatric evaluations, to timely detect patients who may benefit from intense neurocognitive rehabilitation. Future studies, including a complete neuropsychological battery with structural neuroimaging investigations, are necessary to replicate and improve our structural causal model.

Supplementary Information The online version contains supplementary material available at https://doi.org/10.1007/s10072-021-05798-8.

Funding This work was supported by a research grant from Consejo Nacional de Ciencia y Tecnología, Mexico (FORDECYT-PRONACES/49/2021; grant: 11724) to Fernando Daniel Flores Silva, by a research scholarship from the Carlos Slim Foundation (scholarship: R20210029) to Miguel García-Grimshaw, and by a doctoral scholarship (CONACYT-697735) to Amanda Chirino-Pérez. The funders had no role in the study design, data collection, data analysis, data interpretation, writing, or revision of the report.

Data availability All the data supporting our findings are contained within the manuscript. De-identified data to replicate our results will be available to qualified researchers upon written request to the corresponding author.

Code availability Not applicable.

\section{Declarations}

Ethical approval The study was revised and approved by the Instituto Nacional de Ciencias Médicas y Nutrición Salvador Zubirán Ethics and Research Committees (reference: NER-3497-20-21-1). Signed informed consent was obtained from all participants and the procedures about human research were according to the Helsinki declaration.

Conflict of interest The author declare no competing interests. 


\section{References}

1. Tregoning JS, Flight KE, Higham SL et al (2021) Progress of the COVID-19 vaccine effort: viruses, vaccines and variants versus efficacy, effectiveness and escape. Nat Rev Immunol 21:626-636. https://doi.org/10.1038/s41577-021-00592-1

2. Frontera JA, Sabadia S, Lalchan R et al (2021) A prospective study of neurologic disorders in hospitalized COVID-19 patients in New York City. Neurology 96:e575-e586. https://doi.org/10. 1212/WNL.0000000000010979

3. Romero-Sánchez CM, Díaz-Maroto I, Fernández-Díaz E et al (2020) Neurologic manifestations in hospitalized patients with COVID-19: the ALBACOVID registry. Neurology 95:e1060 e1070. https://doi.org/10.1212/WNL.0000000000009937

4. Flores-Silva FD, García-Grimshaw M, Valdés-Ferrer SI et al (2021) Neurologic manifestations in hospitalized patients with COVID-19 in Mexico City. PLoS ONE 16:e0247433. https://doi. org/10.1371/journal.pone.0247433

5. Schou TM, Joca S, Wegener G, Bay-Richter C (2021) Psychiatric and neuropsychiatric sequelae of COVID-19 - a systematic review. Brain Behav Immun 97:328-348. https://doi.org/10. 1016/j.bbi.2021.07.018

6. Garg M, Maralakunte M, Garg S et al (2021) The conundrum of 'Long-COVID-19': a narrative review. Int J Gen Med 14:24912506. https://doi.org/10.2147/IJGM.S316708

7. Huang C, Huang L, Wang Y et al (2021) 6-month consequences of COVID-19 in patients discharged from hospital: a cohort study. Lancet 397:220-232. https://doi.org/10.1016/S0140-6736(20) 32656-8

8. Frontera JA, Yang D, Lewis A et al (2021) A prospective study of long-term outcomes among hospitalized COVID-19 patients with and without neurological complications. J Neurol Sci 426:117486. https://doi.org/10.1016/j.jns.2021.117486

9. Jaywant A, Vanderlind WM, Alexopoulos GS et al (2021) Frequency and profile of objective cognitive deficits in hospitalized patients recovering from COVID-19. Neuropsychopharmacology. https://doi.org/10.1038/s41386-021-00978-8

10. Woo MS, Malsy J, Pöttgen J et al (2020) Frequent neurocognitive deficits after recovery from mild COVID-19. Brain Commun 2:fcaa205. https://doi.org/10.1093/braincomms/fcaa205

11. Mattioli F, Stampatori C, Righetti F et al (2021) Neurological and cognitive sequelae of Covid-19: a four month follow-up. J Neurol. https://doi.org/10.1007/s00415-021-10579-6

12. Almeria M, Cejudo JC, Sotoca J et al (2020) Cognitive profile following COVID-19 infection: Clinical predictors leading to neuropsychological impairment. Brain Behav Immun Health 9:100163. https://doi.org/10.1016/j.bbih.2020.100163

13. Rass V, Beer R, Schiefecker AJ et al (2021) Neurological outcome and quality of life 3 months after COVID-19: a prospective observational cohort study. Eur J Neurol 28:3348-3359. https://doi.org/ 10.1111/ene. 14803

14. World Health Organization (2021) COVID-19 Clinical management: living guidance. https://apps.who.int/iris/bitstream/handle/10665/338882/WHO-2019-nCoV-clinical-2021.1-eng.pdf. Accessed 10 Mar 2021

15. García-Grimshaw M, Chiquete E, Jiménez-Ruiz A et al (2021) Delirium and associated factors in a cohort of hospitalized patients with coronavirus disease 2019. J Acad Consult-Liaison Psychiatry S2667-2960(21):00117-00118. https://doi.org/10. 1016/j.jaclp.2021.06.008

16. RECOVERY Collaborative Group, Horby P, Lim WS et al (2021) Dexamethasone in hospitalized patients with COVID-19. N Engl J Med 384:693-704. https://doi.org/10.1056/NEJMoa2021436

17. Aguilar-Navarro SG, Mimenza-Alvarado AJ, Palacios-García AA et al (2018) Validez y confiabilidad del MoCA (Montreal
Cognitive Assessment) para el tamizaje del deterioro cognoscitivo en méxico. Rev Colomb Psiquiatr 47:237-243. https://doi. org/10.1016/j.rcp.2017.05.003

18. Yeverino-Castro SG, Mejía-Arango S, Mimenza-Alvarado AJ et al (2021) Prevalence and incidence of possible vascular dementia among Mexican older adults: analysis of the Mexican Health and Aging Study. PLoS ONE 16:e0253856. https://doi. org/10.1371/journal.pone.0253856

19. Yamamoto-Furusho JK, Sarmiento-Aguilar A, García-Alanis M et al (2018) Hospital Anxiety and Depression Scale (HADS): validation in Mexican patients with inflammatory bowel disease. Gastroenterol Hepatol 41:477-482. https://doi.org/10. 1016/j.gastrohep.2018.05.009

20. Galindo O, Benjet C, Juárez García F et al (2015) Propiedades psicométricas de la Escala Hospitalaria de Ansiedad y Depresión (HADS) en una población de pacientes oncológicos mexicanos. Salud Ment 38:253-258. https://doi.org/10.17711/ SM.0185-3325.2015.035

21. Durón Figueroa R (2019) Adaptación de la Lista Checable de Trastorno por Estrés Postraumático para DSM-5 en Población Mexicana. Acta Investig Psicológica 9:26-36. https://doi.org/ 10.22201/fpsi.20074719e.2019.1.03

22. Charlson ME, Pompei P, Ales KL, MacKenzie CR (1987) A new method of classifying prognostic comorbidity in longitudinal studies: development and validation. J Chronic Dis 40:373-383. https://doi.org/10.1016/0021-9681(87)90171-8

23. Definition Task Force ARDS, Ranieri VM, Rubenfeld GD et al (2012) Acute respiratory distress syndrome: the Berlin Definition. JAMA 307:2526-2533. https://doi.org/10.1001/jama.2012. 5669

24. Vincent JL, Moreno R, Takala J et al (1996) The SOFA (Sepsis-related Organ Failure Assessment) score to describe organ dysfunction/failure. On behalf of the Working Group on SepsisRelated Problems of the European Society of Intensive Care Medicine. Intensive Care Med 22:707-710. https://doi.org/10. 1007/BF01709751

25. Zhao Q, Meng M, Kumar R et al (2020) Lymphopenia is associated with severe coronavirus disease 2019 (COVID-19) infections: a systemic review and meta-analysis. Int J Infect Dis 96:131-135. https://doi.org/10.1016/j.ijid.2020.04.086

26. Osuchowski MF, Winkler MS, Skirecki T et al (2021) The COVID-19 puzzle: deciphering pathophysiology and phenotypes of a new disease entity. Lancet Respir Med 9:622-642. https://doi. org/10.1016/S2213-2600(21)00218-6

27. Vabret N, Britton GJ, Gruber C et al (2020) Immunology of COVID-19: current state of the science. Immunity 52:910-941. https://doi.org/10.1016/j.immuni.2020.05.002

28. Mardia K (1985) Mardia's test of multinormality. In: Johnson NL, Kotz S, Read C (eds) Encyclopaedia of statistics. Wiley, New York

29. Bollen KA, Stine RA (1992) Bootstrapping goodness-of-fit measures in structural equation models. Sociol Methods Res 21:205229. https://doi.org/10.1177/0049124192021002004

30. Bruijnen CJWH, Dijkstra BAG, Walvoort SJW et al (2020) Psychometric properties of the Montreal Cognitive Assessment (MoCA) in healthy participants aged 18-70. Int J Psychiatry Clin Pract 24:293-300. https://doi.org/10.1080/13651501.2020.17463 48

31. Silay K, Yalcin A, Akinci S et al (2017) Charlson Comorbidity Index, inappropriate medication use and cognitive impairment : Bermuda Triangle. Wien Klin Wochenschr 129:799-804. https:// doi.org/10.1007/s00508-017-1253-4

32. Wei MY, Levine DA, Zahodne LB et al (2020) Multimorbidity and cognitive decline over 14 years in older Americans. J Gerontol A Biol Sci Med Sci 75:1206-1213. https://doi.org/10.1093/gerona/ glz147 
33. Mikkelsen ME, Christie JD, Lanken PN et al (2012) The adult respiratory distress syndrome cognitive outcomes study: long-term neuropsychological function in survivors of acute lung injury. Am J Respir Crit Care Med 185:1307-1315. https://doi.org/10.1164/ rccm.201111-2025OC

34. Jackson JC, Hart RP, Gordon SM et al (2003) Six-month neuropsychological outcome of medical intensive care unit patients. Crit Care Med 31:1226-1234. https://doi.org/10.1097/01.CCM. 0000059996.30263 .94

35. Shah FA, Girard TD, Yende S (2017) Limiting sedation for patients with acute respiratory distress syndrome - time to wake up. Curr Opin Crit Care 23:45-51. https://doi.org/10.1097/MCC. 0000000000000382

36. Pun BT, Badenes R, Heras La Calle G et al (2021) Prevalence and risk factors for delirium in critically ill patients with COVID19 (COVID-D): a multicentre cohort study. Lancet Respir Med 9:239-250. https://doi.org/10.1016/S2213-2600(20)30552-X

37. Goldberg TE, Chen C, Wang Y et al (2020) Association of delirium with long-term cognitive decline: a meta-analysis. JAMA Neurol 77:1373-1381. https://doi.org/10.1001/jamaneurol.2020. 2273

38. Khan SH, Lindroth H, Perkins AJ et al (2020) Delirium incidence, duration, and severity in critically ill patients with coronavirus disease 2019. Crit Care Explor 2:e0290. https://doi.org/10.1097/ CCE.0000000000000290

39. Ticinesi A, Cerundolo N, Parise A et al (2020) Delirium in COVID-19: epidemiology and clinical correlations in a large group of patients admitted to an academic hospital. Aging Clin Exp Res 32:2159-2166. https://doi.org/10.1007/ s40520-020-01699-6

40. Mcloughlin BC, Miles A, Webb TE et al (2020) Functional and cognitive outcomes after COVID-19 delirium. Eur Geriatr Med 11:857-862. https://doi.org/10.1007/s41999-020-00353-8

41. Carlson CG, Huang DT (2013) The Adult Respiratory Distress Syndrome Cognitive Outcomes Study: long-term neuropsychological function in survivors of acute lung injury. Crit Care 17:317. https://doi.org/10.1186/cc12709

42. Miskowiak KW, Johnsen S, Sattler SM et al (2021) Cognitive impairments four months after COVID-19 hospital discharge: pattern, severity and association with illness variables. Eur Neuropsychopharmacol 46:39-48. https://doi.org/10.1016/j.euron euro.2021.03.019

43. Hopkins RO, Gale SD, Weaver LK (2006) Brain atrophy and cognitive impairment in survivors of acute respiratory distress syndrome. Brain Inj 20:263-271. https://doi.org/10.1080/02699 050500488199

44. Hota SK, Barhwal K, Singh SB, Ilavazhagan G (2007) Differential temporal response of hippocampus, cortex and cerebellum to hypobaric hypoxia: a biochemical approach. Neurochem Int 51:384-390. https://doi.org/10.1016/j.neuint.2007.04.003
45. Lu Y, Li X, Geng D et al (2020) Cerebral micro-structural changes in COVID-19 patients - an MRI-based 3-month follow-up Study. EClinicalMedicine 25:100484. https://doi.org/10.1016/j.eclinm. 2020.100484

46. Hampshire A, Trender W, Chamberlain SR et al (2021) Cognitive deficits in people who have recovered from COVID-19. EClinicalMedicine 39:101044. https://doi.org/10.1016/j.eclinm.2021. 101044

47. Mazza MG, Palladini M, De Lorenzo R et al (2021) Persistent psychopathology and neurocognitive impairment in COVID-19 survivors: Effect of inflammatory biomarkers at three-month follow-up. Brain Behav Immun 94:138-147. https://doi.org/10. 1016/j.bbi.2021.02.021

48. Ferrucci R, Dini M, Groppo E et al (2021) Long-lasting cognitive abnormalities after COVID-19. Brain Sci 11:235. https://doi.org/ 10.3390/brainsci11020235

49. Nalbandian A, Sehgal K, Gupta A et al (2021) Post-acute COVID19 syndrome. Nat Med 27:601-615. https://doi.org/10.1038/ s41591-021-01283-z

50. Zhou H, Lu S, Chen J et al (2020) The landscape of cognitive function in recovered COVID-19 patients. J Psychiatr Res 129:98102. https://doi.org/10.1016/j.jpsychires.2020.06.022

51. Qin $\mathrm{Y}, \mathrm{Wu} \mathrm{J}$, Chen $\mathrm{T}$ et al (2021) Long-term microstructure and cerebral blood flow changes in patients recovered from COVID-19 without neurological manifestations. J Clin Invest 131:147329. https://doi.org/10.1172/JCI147329

52. Erickson MA, Rhea EM, Knopp RC, Banks WA (2021) Interactions of SARS-CoV-2 with the blood-brain barrier. Int J Mol Sci 22:2681. https://doi.org/10.3390/ijms22052681

53. Annane D, Sharshar T (2015) Cognitive decline after sepsis. Lancet Respir Med 3:61-69. https://doi.org/10.1016/S2213-2600(14) 70246-2

54. Janiri D, Carfì A, Kotzalidis GD et al (2021) Posttraumatic stress disorder in patients after severe COVID-19 infection. JAMA Psychiat 78:567-569. https://doi.org/10.1001/jamapsychiatry.2021. 0109

55. Del Brutto OH, Wu S, Mera RM et al (2021) Cognitive decline among individuals with history of mild symptomatic SARSCoV-2 infection: a longitudinal prospective study nested to a population cohort. Eur J Neurol 28:3245-3253. https://doi.org/ 10.1111/ene. 14775

56. Blomberg B, Mohn KG-I, Brokstad KA et al (2021) Long COVID in a prospective cohort of home-isolated patients. Nat Med 27:1607-1613. https://doi.org/10.1038/s41591-021-01433-3

Publisher's note Springer Nature remains neutral with regard to jurisdictional claims in published maps and institutional affiliations. 


\section{Authors and Affiliations}

Miguel García-Grimshaw ${ }^{1}$ (1) - Amanda Chirino-Pérez ${ }^{2}$ (1) - Fernando Daniel Flores-Silva ${ }^{1}$ (1) Sergio Iván Valdés-Ferrer ${ }^{1,3}$ [ $\cdot$ María de los Ángeles Vargas-Martínez ${ }^{1} \cdot$ Ana Itiel Jiménez-Ávila ${ }^{1}$. Oswaldo Alan Chávez-Martínez ${ }^{1}$ - Enrique Manuel Ramos-Galicia ${ }^{4}$. Osvaldo Alexis Marché-Fernández ${ }^{1}$. Martha Fernanda Ramírez-Carrillo ${ }^{4}$ - Samara Lissete Grajeda-González ${ }^{1}$. Marco Eduardo Ramírez-Jiménez ${ }^{1}$. Emma Adriana Chávez-Manzanera ${ }^{5}\left(\mathbb{D}^{\circ} \cdot\right.$ María Teresa Tusié-Luna $^{6,7}$ (1) Ana Ochoa-Guzmán ${ }^{6}$. Carlos Cantú-Brito ${ }^{1}$ (1) . Juan Fernandez-Ruiz ${ }^{2,8}$ (1) Erwin Chiquete ${ }^{1}$ (1)

Juan Fernandez-Ruiz jfr@unam.mx

Erwin Chiquete erwin.chiquetea@incmnsz.mx

Miguel García-Grimshaw miguelgrimshaw@gmail.com

Amanda Chirino-Pérez amandachirino@gmail.com

Fernando Daniel Flores-Silva ferfs98@gmail.com

Sergio Iván Valdés-Ferrer sergio.valdesf@incmnsz.mx

María de los Ángeles Vargas-Martínez avargasmartnez@gmail.com

Ana Itiel Jiménez-Ávila anita.jimenezz.aj@gmail.com

Oswaldo Alan Chávez-Martínez alanchavezhjm@gmail.com

Enrique Manuel Ramos-Galicia manuel_ram95@hotmail.com

Osvaldo Alexis Marché-Fernández osvalmarche@gmail.com

Martha Fernanda Ramírez-Carrillo martha.ramirezc1@gmail.com

Samara Lissete Grajeda-González samara-grajeda@hotmail.com

Marco Eduardo Ramírez-Jiménez me.ramirezjimenez@gmail.com
Emma Adriana Chávez-Manzanera emanzanera@hotmail.com

María Teresa Tusié-Luna mttusie@gmail.com

Ana Ochoa-Guzmán aochoaguzman@yahoo.com.mx

Carlos Cantú-Brito carloscantu_brito@hotmail.com

1 Department of Neurology and Psychiatry, Instituto Nacional de Ciencias Médicas y Nutrición Salvador Zubirán, Mexico City, México

2 Neuropsychology Laboratory, Department of Physiology, Faculty of Medicine, National Autonomous University of Mexico, Mexico City, Mexico

3 Feinstein Institutes for Medical Research, Manhasset, NY, USA

4 Faculty of Psychology, National Autonomous University of Mexico, Mexico City, Mexico

5 Department of Endocrinology and Metabolism, Instituto Nacional de Ciencias Médicas y Nutrición Salvador Zubirán, Mexico City, Mexico

6 Unidad de Biología Molecular y Medicina Genómica, Instituto Nacional de Ciencias Médicas y Nutrición Salvador Zubirán, Mexico City, Mexico

7 Instituto de Investigaciones Biomédicas, Universidad Nacional Autónoma de México, Mexico City, Mexico

8 Faculty of Psychology, Universidad Veracruzana, Veracruz, Mexico 